\title{
The effects of methyl eugenol, cue lure and plant essential oils in rubber foam dispenser for controlling Bactrocera dorsalis and Zeugodacus cucurbitae
}

\author{
Most Mottakina Akter¹, Keum Theary¹, Ekwipoo Kalkornsurapranee², Chandra Shekhar Prabhakar ${ }^{3}$, \\ Narit Thaochan ${ }^{*}$ \\ ${ }^{1}$ Agricultural Innovation and Management Division, Faculty of Natural Resources, Prince of Songkla University, Songkhla, \\ Thailand \\ ${ }^{2}$ Department of Materials Science and Technology, Faculty of Science, Prince of Songkla University, Songkhla, Thailand \\ ${ }^{3}$ Department of Entomology, Veer Kunwar Singh College of Agriculture, Bihar Agriculture University, Dumraon-802136, \\ Buxar, Bihar, India
}

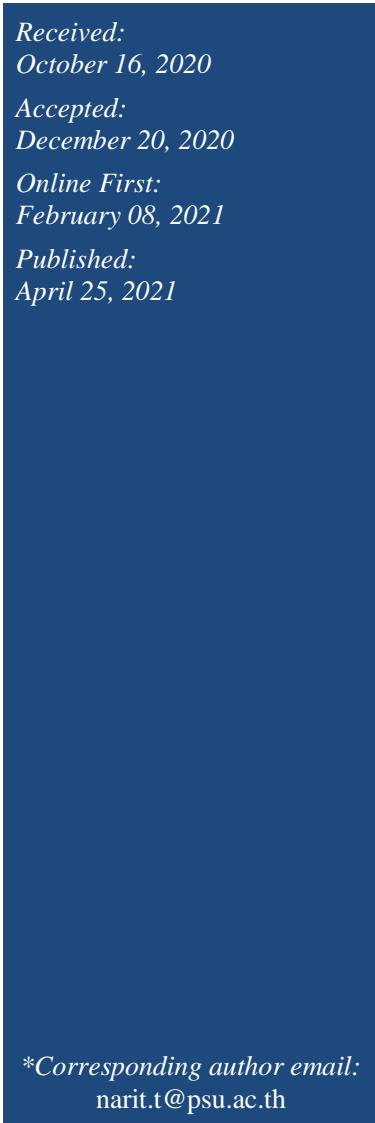

narit.t@psu.ac.th

\begin{abstract}
Bactrocera dorsalis and Zeugodacus cucurbitae are the most economically important pests of fruits and vegetables production. Male lures like, methyl eugenol (ME) and cue lure $(\mathrm{CL})$ in conjunction with insecticides are commercially used to attract and kill these pests. We investigated, herein, the application of plant essential oils (basil, clove, citronella, and eucalyptus) with ME and CL for both toxicity and attraction. Through the use of rubber foam as a base, basil oil (BO) was found to be highly toxic to $B$. dorsalis and Z. cucurbitae, and was evaluated for potency with ME and CL as effective attraction mechanisms. Our results found $15.7-20.3 \%$ of attraction for $B$. dorsalis using ME, separately or mixed with basil oil. The separate CL found $20.3 \%$ while the $\mathrm{ME}+\mathrm{CL}$ mixture provided $21.5 \%$ attraction for $Z$. cucurbitae. In field tests, a greater number of $B$. dorsalis were trapped with ME alone (20.4 fly/trap/day, FTD), whereas the attraction of the ME and $\mathrm{BO}$ mixture was relatively low. For Z. cucurbitae, ME+CL (0.1-0.7 FTD) and CL (0.2-0.6 FTD) trapped more flies than mixtures combining BO, suggesting that combinations of $\mathrm{BO}$ with $\mathrm{ME}$ in rubber foam bases may be a viable alternative for the control $B$. dorsalis but not for $Z$. cucurbitae.
\end{abstract}

Keywords: Para-pheromone, Mixed lure, Plant essential oils, Rubber foam, Attract and kill, Tephritid fruit fly

\section{How to cite this:}

Akter MM, Theary K, Kalkornsurapranee E, Prabhakar CS and Thaochan N, 2021. The effects of methyl eugenol, cue lure and plant essential oils in rubber foam dispenser for controlling Bactrocera dorsalis and Zeugodacus cucurbitae. Asian J. Agric. Biol. 2021(2): 202010530. DOI: https://doi.org/10.35495/ajab.2020.10.530

This is an Open Access article distributed under the terms of the Creative Commons Attribution 3.0 License. (https://creativecommons.org/licenses/by/3.0), which permits unrestricted use, distribution, and reproduction in any medium, provided the original work is properly cited.

\section{Introduction}

The Oriental fruit fly, Bactrocera dorsalis (Hendel), and the melon fly, Zeugodacus cucurbitae (Coquillett) of the family Tephritidae are the most destructive fruit fly species worldwide, particularly in tropic and subtropic regions (Vargas et al., 2000; Prabhakar et al., 2012a; Qin et al., 2018). They cause direct losses to 
fruit and vegetable crops in the field, as well as indirect losses related to the restrictions on the export of fruits and vegetables due to bio-security concerns of these species in various parts of the world (Aketarawong et al., 2014). These pests have been managed primarily via food bait, bait sprays, para-pheromones, and insecticides for the past century. To control tephritid flies the 'lure and kill' approach, based on food attractants or para-pheromones mixed with a killing agent, have been more recently employed as part of the Integrated Pest Management (IPM) plan (Canale et al., 2013). Due to the increasing demand for organic foods, successful practices of the 'lure and kill' system within the guidelines of an organic farming system are needed.

Male attractants, such as methyl eugenol (ME) and the cue lure (CL) have been successfully used over the past few decades for the detection and control of fruit flies; namely, the oriental fruit fly and melon fruit fly. To date, no fruit fly species have been found to react with combinations of ME and CL, as their responses were deemed to be connected to special antennal receptor sites (Metcalf and Metcalf, 1992). Where such fruit flies prevail, several experiments have been conducted to evaluate the effectiveness of baits mixed with $\mathrm{ME}$ and $\mathrm{CL}$ for recognition and control of fruit flies. The careful and creative mixing of lures could lessen the number of traps in such areas, as well as reduce the number of laborers necessary to maintain them (Royer and Mayer, 2017).

Excessive use of synthetic pesticides is associated with many environmental problems, such as insecticide resistance, lethality to other organisms, persistence in soil and food, and ecological pollution (Desneux et al., 2007), and are not suitable for organic cultivation. These factors create an urgent need to switch over to natural products that are environmentally friendly, less hazardous to other organisms, and suitable for organic farming. Plant extracts containing insecticidal properties against tephritid fruit flies could be potential alternatives to synthetic insecticides (Benelli et al., 2012), as they are more ecologically friendly than synthetic insecticides (Isman and Machial, 2006). Many plant families contain sufficient amounts of essential oils (EOs) that are toxic to insect pests; such as Apiaceae, Asteraceae, Fabaceae, Lamiaceae, Lauraceae, Poaceae, Myrtaceae, Rutaceae, and Zingiberaceae (Maggi and Benelli, 2018). In our study, we tested four plant essential oils: basil oil; clove oil; citronella oil; and eucalyptus oil, known to possess several volatile bioactive chemicals. Chang et al. (2009, 2013) investigated the insecticidal properties of basil oil (Ocimum spp. Lamiaceae) in fruit flies, which produced $99 \%$ adult mortality in Callosobruchus maculatus (Fabricius) compared to 0 $\%$ for the control. In addition to mortality, basil oil was also found to decrease oviposition and in-egg hatchings (Kéita et al., 2000). Other broadly used EOs include clove oil (Eugenia caryophyllata Spreng, Myrtaceae) and Citronella oil (Cymbopogon nardus L. Poaceae). Arancibia et al. (2013) noted the effects of 3 $\%$ clove and citronella oils with a soy protein-lignin film incorporation on the time of knockdown of Mediterranean fruit flies (Ceratitis capitata) (Wiedemann). Their results showed that vapors emitted from the clove film caused the mortality of 40 $\%$ of the flies after four hours of exposure, which increased to $90 \%$ after twenty hours. They determined that the active ingredients of citronella oil may not have released effectively from the film, and, therefore, suggested that citronella oil may be more effective in raw form. In further studies, Ilyas et al. (2017) found that eucalyptus leaf extracts (Eucalyptus camaldulensis Dehnh., Myrtaceae) demonstrated satisfactory repellence oviposition inhibition of peach fruit flies; and Rossi and Palacios (2015) determined that $\mathrm{LC}_{50}$ of Eucalyptus cinerea F.Muell. ex Benth. at $5.5 \mathrm{mg} / \mathrm{dm}^{3}$ through fumigation assay, killed flies within 15 minutes.

Among tephritid flies, the Mediterranean fruit fly, $C$. capitata, is the most studied species interacting with essential oils (López et al., 2011), whereas B. dorsalis and $Z$. cucurbitae investigations with EOs have been less examined (Chang et al., 2009, 2013). Many types of dispensers; such as wood, cardboard, polymer, cotton, lure plug, and wafers have been used with different chemicals for the attraction of fruit flies worldwide. In the present study, we applied a novel approach incorporating rubber foam from natural latex which slowly released small amounts of parapheromone that can remain active for 90 to 120 days (Malee, 2019).

The goal of the present study, therefore, was to screen plant essential oils with ME and/or CL in rubber foam lures against $B$. dorsalis and $Z$. cucurbitae, to assess the attractiveness of the single and mixed lure methods under both laboratory and field conditions, and to ultimately develop an alternative 'lure and kill' system sustainable in organic farming systems. 


\section{Material and Methods}

\section{Insect rearing}

Insects used in this study were reared from infested guava (Psidium guajava) and luffa (Luffa acutangular) fruit collected from the agricultural fields of Prince of Songkla University, Hat Yai district, Songkhla province, Thailand (latitude and longitude: $7.004658^{\circ} \mathrm{N}, 100.503350^{\circ} \mathrm{E}$ ). The infested fruit with larvae was kept in a transparent plastic box $(25 \times 20 \times 15 \mathrm{~cm})$ with a perforated lid for air ventilation, filled with a layer $(1 \mathrm{~cm})$ of sterile sawdust (autoclaved and dried) at the bottom of the box as a medium for pupation. Upon the adult fly's emergence, they were transferred to another insect-rearing cage $(30 \times 30 \times 30 \mathrm{~cm})$. To maintain the growth of the adult flies, cube sugar, yeast hydrolysate, and water $a d$ libitum were provided. Adult $B$. dorsalis and $Z$. cucurbitae were identified from the adult flies ten days post-emergence based on the morphological characteristics described by Prabhakar et al. (2012b) and Drew and Romig (2013).

Pure cultures of $B$. dorsalis and Z. cucurbitae were further maintained in separate insect-rearing cages as described above. Laboratory conditions for rearing $B$. dorsalis and Z. cucurbitae were maintained at 12:12 hours of light and dark periods at $75-80 \%$ relative humidity (RH) and at temperatures of $27 \pm 2{ }^{\circ} \mathrm{C}$. To maintain a continuous stock of experimental flies, adult flies of both sexes of the same species were kept in the same cage for mating and oviposition. Within our experiments, only 10-15 day-old adult flies were used from the stock culture.

\section{Essential oils}

Commercial grade of basil (Ocimum spp.), clove (Eugenia spp.), citronella (Cymbopogon spp.), and eucalyptus (Eucalyptus spp.) essential oils were purchased from Jiangxi Yisenyuan Plant Species Co., Ltd; China.

\section{Rubber foam preparation}

Para rubber foam was prepared using a modification of Dunlop's technique (Calvert, 1982; Malee, 2019). High ammonia concentrated natural latex $60 \%$ (167 g) was first stirred for five minutes to remove the preservative. Other chemical ingredients included potassium-oleate solution $20 \%$ (10 g), Sulphur dispersion $50 \%$ (5 g), zinc-N-diethyldithiocarbamate dispersion $50 \%$ (2 g), zinc-2-mercaptobenzothiazole dispersion $50 \%(2 \mathrm{~g})$, butylated hydroxytoluene $50 \%$
(2 g), diphenyl guanidine dispersion $30 \%$ (20 g), zinc oxide dispersion $50 \% \quad(20 \mathrm{~g})$, and sodium silicofluoride dispersion $20 \%(10 \mathrm{~g})$. Each of the chemicals was added into the latex within ten minutes under continuous stirring. The formed latex was poured into molds and then vulcanized in a hot air oven at $70{ }^{\circ} \mathrm{C}$ for three hours. The resulting pararubber foam was then dipped into compounded latex in which to produce a thin film over the foam and cut into $2 \times 5 \mathrm{~cm}$ pieces for use as dispensers. The para rubber foam dispensers were kept at the ambient temperature before bioassay.

Each plant essential oil, $300 \mu 1$ of basil oil, clove oil, citronella oil, and eucalyptus oil, was loaded into separate rubber foam dispensers $(2 \times 5 \mathrm{~cm})$ using individual $1 \mathrm{ml}$ syringes. The rubber foam dispensers with essential oil, as well as a control (without essential oil) were weathered in a natural environment for $0,15,30$, and 45 days. Four foam dispensers were selected from each group on each respective day for experimental investigation.

\section{Fumigation toxicity bioassay}

In conducting the bioassay, ten $B$. dorsalis and $Z$. cucurbitae adult flies (five male and five female; 10 15 days old) were kept in a clear plastic container (750 $\mathrm{ml}$ ) with some minute holes in the lid. A rubber foam dispenser with a specified weathering period $(0,15$, 30 , and 45 days) of essential oil was placed on the bottom of the container to expose the adult flies for a maximum of 72 hours. Yeast, sugar, and water-soaked cotton were provided as food. The experiment was conducted in a Completely Randomized Design (CRD) with four replications for B. dorsalis and three replications for $Z$. cucurbitae. Mortality was observed at 1, 6, 24, 48, and 72 hours after exposure. The plant essential oil with the highest percentage of mortality was selected for the next experiment.

\section{The attraction test of the $B$. dorsalis and $Z$. cucurbitae to lures}

Methyl eugenol (ME) (4-allyl-1,2-dimethoxybenzene, CAS 93-15-2) and the cue lures (CL) [4-(pacetoxyphenyl)-2-butanone, CAS 3572-06-3] were supplied by Sigma-Aldrich, St. Louis, MO, USA. One hundred $\mu 1$ of each single ME or CL lure was injected into a rubber foam dispenser. Combinations of the lure (50 $\mu \mathrm{l}$ of ME+CL injected in the same spot and $50 \mu \mathrm{l}$ of $\mathrm{ME} / \mathrm{CL}$ injected in a different spot) were injected into a rubber foam dispenser. The pore size of the foam consisted of small holes or pockets in which to retain 
the liquid chemicals. When we injected both lures (ME and CL) at separate points, they were not mixed but volatilized. Lures injected at the same spot in the dispenser were also volatilized. The experiment followed a Completely Randomized Design (CRD) with five treatments: ME; CL; ME+CL; ME/CL; and the control (foam without chemicals) with four replications of each treatment. After being weathered outside in a natural environment for $0,15,30$, or 45 days; ten males $B$. dorsalis and ten male $Z$. cucurbitae for each treatment were released in a gauge cage $(30 \times 30 \times 30 \mathrm{~cm})$. Yeast, sugar, and water ad libitum were supplied. After that, a foam dispenser (previously prepared with $100 \mu \mathrm{l}$ of the lure) was put above the cage for one hour. The numbers of attracted flies were recorded for each species and calculated as a percentage of attraction.

\section{Attraction test of fruit flies with lures and essential oil at the same point on rubber foam dispensers}

Similar amounts of ME, CL, ME+CL, and ME/CL were prepared as described above. Three hundred $\mu \mathrm{l}$ of basil oil was injected in a foam dispenser at the same spot where the lures were previously loaded. For $\mathrm{ME} / \mathrm{CL}$, half of the $300 \mu \mathrm{l}$ of basil oil was separately injected in the same spot as the ME and CL in the rubber foam dispenser. This experiment was also conducted in a Completely Randomized Design (CRD) with five treatments: $\mathrm{ME}+\mathrm{BO}$; $\mathrm{CL}+\mathrm{BO}$; $\mathrm{ME}+\mathrm{CL}+\mathrm{BO} ; \mathrm{ME} / \mathrm{CL}+\mathrm{BO}$; and the control with four replications for each treatment. The foam dispensers were then were weathered outside in the natural environment for $0,15,30$, and 45 days. After that, ten male $B$. dorsalis and ten male $Z$. cucurbitae were released in a gauge cage $(30 \times 30 \times 30 \mathrm{~cm})$. The foam dispenser was kept above the cage for one hour. The percentage of attractiveness was calculated for each species within each treatment.

\section{Attraction test of flies with lures and essential oil at different points on rubber foam dispensers}

Equal treatment amounts of ME, CL, ME+CL, and $\mathrm{ME} / \mathrm{CL}$ were prepared as described above. Three hundred $\mu \mathrm{l}$ of basil oil was injected in the foam dispensers at a different point from where the lures were previously loaded. The experiment was conducted in Completely Randomized Design (CRD) for the five treatments; $\mathrm{CL} / \mathrm{BO}, \mathrm{ME} / \mathrm{BO}, \mathrm{ME}+\mathrm{CL} / \mathrm{BO}$, $\mathrm{ME} / \mathrm{CL} / \mathrm{BO}$, and the control (foam without chemicals) with four replications per treatment. Again, the foam dispensers were weathered in the natural environment for $0,15,30$, or 45 days; a net cage was prepared, and ten male $B$. dorsalis and ten male $Z$. cucurbitae were released. The foam dispenser was placed above the case for one hour, and the number of flies was recorded for each species for each treatment.

\section{The efficiency of mixed lures and essential oils in field conditions}

Field experiments were conducted in the three sapodilla orchards located in Koh Yor, Songkhla province, Thailand: Orchard 1, $7.152179{ }^{\circ} \mathrm{N}$ $100.541570{ }^{\circ} \mathrm{W}(\sim 0.32 \mathrm{ha})$; Orchard 2, $7.157172{ }^{\circ} \mathrm{N}-$ $100.536131^{\circ} \mathrm{W}(\sim 0.22 \mathrm{ha})$; and Orchard 3, 7.152701 ${ }^{\circ} \mathrm{N}-100.532129{ }^{\circ} \mathrm{W}(\sim 0.20$ ha. The orchards were at distances around 2-3 km apart. Seven selected treatments (ME, CL, ME+CL, ME/BO, CL/BO, $\mathrm{ME}+\mathrm{CL} / \mathrm{BO}$, and the control underwent three replications each, totaling 21 traps per orchard. The lure $(100 \mu \mathrm{l})$ and the essential oil $(300 \mu \mathrm{l})$ were injected in the foam dispenser similar to the previous experiments. The foam lures were then tied into the lids of the clear plastic cup traps $(750 \mathrm{ml})$ with aluminum wire. Four lateral holes, $10 \mathrm{~mm}$ in diameter, were made at the upper sidewall of the trap as an entry route for flies. The base portion of the trap container was wrapped with a yellow trap to attract flies.

The experiment design was conducted in Randomized Completely Block Design (RCBD) with seven treatments and three replications (orchards). The traps with only lures (ME, CL, and ME+CL) were prepared with $200 \mathrm{ml}$ of $20 \%$ propylene glycol to attract flies. Propylene glycol was not added into the lures with basil oil (ME/BO, CL/BO, and ME+CL/BO), as an additional killing agent was not needed. The traps were installed under the trees at $150 \mathrm{~cm}$ above ground and at a minimum distance of $10 \mathrm{~m}$.

Traps were initially set on 15 Aug 2019, and the first observation began on 1 Sep 2019, which continued at 15-day intervals until the end of the experiment on 2 Feb 2020. Lures were replaced monthly throughout the experiment. Lastly, species were separated and identified in a laboratory setting based on the morphological characteristics under a Leica S8APO stereo zoom microscope (Wetzlar, Germany) according to Drew and Romig, 2013. Meteorological data; such as temperature, relative humidity, and rainfall were also recorded for their various effects upon the experiments' success.

\section{Statistical analysis}

Percentages of mortality and attraction were generated 
under laboratory conditions, transformed by arcsine and square root analyses. Data were subjected to one-way ANOVA and means comparisons were made via Tukey's Honestly Significant Difference Test $(\alpha=$ 0.05 ). The number of trapped flies within the field conditions was converted to flies per trap per day (FTD), transformed by $\log (\mathrm{X}+0.5)$. Repeatedmeasure ANOVA tests were performed with time as a recurring factor to test for different numbers of FTD between treatments. All analyses were performed using an SPSS 11.0 program for Windows (SPSS, 2001).

\section{Results}

\section{Mortality assay for B. dorsalis and Z. cucurbitae}

For $B$. dorsalis, the percentage of cumulative mortality of adult fruit flies varied significantly with each essential oil. Eucalyptus oil outperformed the other oils within 12 hours of exposure, in which $100 \%$ mortality was observed $\left(\mathrm{F}_{4,20}=58.412 ; p<0.01\right)$ (Figure 1A). In contrast, other EOs displayed continued activity 24 to 72 hours after exposure. The observations on day 15 , after 72 hours, found that basil oil produced $95.0 \pm 2.9 \%$ fly mortality, followed by clove oil with $87.5 \pm 2.5 \%$ mortality with a significant variation $\left(\mathrm{F}_{4,20}=276.964 ; p<0.001\right)$ (Figure 1B). The other EOs did not show satisfactory effectiveness. On day 30 , the basil and clove oils were moderately active with $72.5 \pm 2.5 \%$ and $67.5 \pm 10.3 \%$, respectfully, amid further reduced effectiveness from the other essential oils (Figure 1C). Lastly, on day 45, none of the oils remained effective (Figure 1D). And, naturally, no mortality occurred within the control treatment.
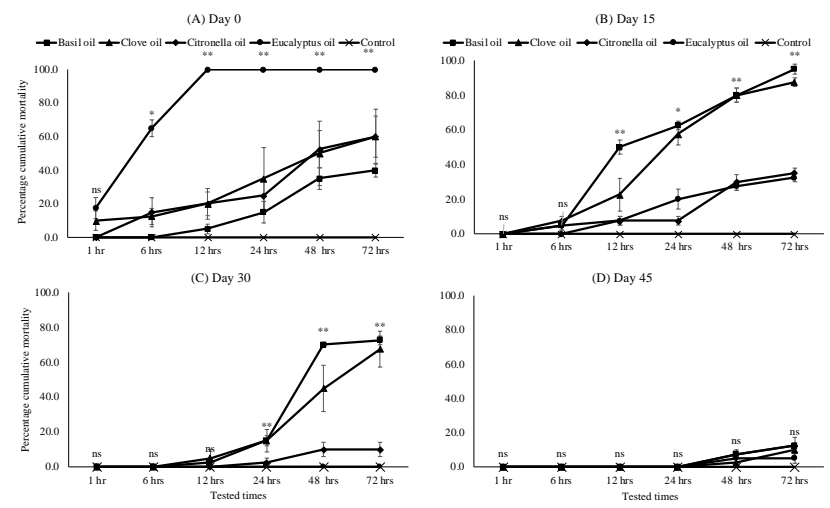

Figure-1. Cumulative mortalities of adult Bactrocera dorsalis from rubber foam injected with a plant essential oil (basil oil, clove oil, citronella oil, eucalyptus oil, and the control); weathered for $(A) 0$, (B) 15, (C) 30, and (D) 45 days.
For Z. cucurbitae, the cumulative percentage mortality of adult flies exposed to the essential oils was again significant. Eucalyptus oil again proved superior with $100 \%$ mortality within the first 12 hours of exposure (Figure 2A). By contrast, the other oils were far less effective. On day 15, after 72 hours, basil oil and clove oil produced $76.7 \pm 6.7 \%$ and $40.0 \pm 11.5 \%$ mortality, respectively $\left(\mathrm{F}_{4,15}=16.562 ; p<0.01\right)$ (Figure $\left.2 \mathrm{~B}\right)$. On day 30; basil, clove, and citronella oils were found to produce minimum mortality, yet did not differ significantly (Figure 2C). On the last day of observation, Day 45, none of the oils were effective (Figure 2D). Again, no dead flies were not found in the control.
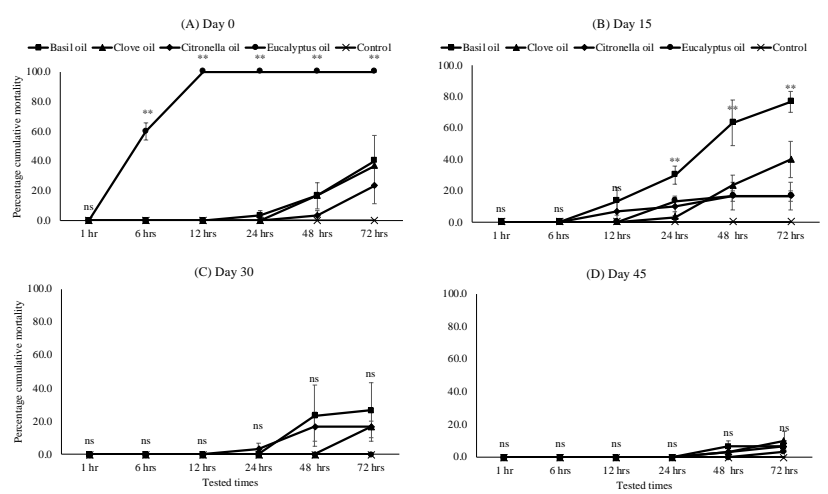

Figure-2. Cumulative mortalities of adult Zeugodacus cucurbitae from rubber foam injected with plant essential oils (basil oil, clove oil, citronella oil, eucalyptus oil, and the control), weathered for (A) 0 , (B) 15, (C) 30, or (D) 45 days. The attraction of adult male of $B$. dorsalis and $Z$. cucurbitae to methyl eugenol with a single or mixed cue lure

The percentages of attraction of $B$. dorsalis by the single (ME) and mixed lure (ME+CL and ME/CL) on Day 0 proved significantly different $\left(\mathrm{F}_{4,20}=34.627 ; p<\right.$ $0.001)$. The highest percentage of attractiveness was obtained with ME at $32.5 \pm 4.8 \%$. On day 15 through 45 , the percentages across all treatments were not significantly different. For Z cucurbitae, the mixed lure $(\mathrm{ME}+\mathrm{CL})$ gave higher percentages of attraction on Days 0 and 15 than those of the cue lure (CL). On Days 30 to 45 , the percentages of attraction for both the single (CL) and mixed lures (ME+CL and ME/CL) were not significantly different (Table 1). 
Attraction of adult male $B$. dorsalis and $Z$. cucurbitae to rubber foam injected with basil oil in the same spot with methyl eugenol and a single cue lure or mixed lure

The percentages of attraction for both $B$. dorsalis and $Z$. cucurbitae decreased when all lures were mixed with basil oil. At Day 0, the percentages of attraction for both species in all treatments ranged from 0.0-8.3 $\%$. For $B$. dorsalis, the percentages of attraction from days 15-45 ranged from 13.3-28.3\%, whereas for $Z$. cucurbitae, the percentages of attraction were less than $15.0 \%$ (Table 2).

Boxplot analyses of the attraction levels were performed from Day 0 to Day 45. The single ME, $\mathrm{ME}+\mathrm{CL}$, and $\mathrm{ME} / \mathrm{CL}$ with and without $\mathrm{BO}$ were not significantly different, ranging from 15.5-20.3\%; whereas the single $\mathrm{CL}$ and $\mathrm{CL}$ with and without $\mathrm{BO}$ did not attract this fly species (Figure 3A).

The attraction of adult male $B$. dorsalis and $Z$. cucurbitae to rubber foam injected with basil oil in a different spot with methyl eugenol and a single cue lure or mixed lure

The percentages of attraction for both $B$. dorsalis and $Z$. cucurbitae decreased when all lures were mixed with basil oil at a different spot in the rubber foam. At Day 0 , the percentages of attraction for both species in all treatments were less than $20.0 \%$. For B. dorsalis, the percentages of attraction from Days 15-45 were similar to the blended basil oil and lure, which ranged from 10.0-28.3\%. For Z. cucurbitae, the percentages of attraction were similar to those of the previous experiment (Table 3).

Boxplot analyses of attraction from Day 0 to Day 45 were performed. The ME+CL $(21.5 \pm 2.3 \%)$ and $\mathrm{CL}$ $(20.5 \pm 1.5 \%)$ attracted more flies than those mixed with BO (6.7 -13.3\%) (Figure 3B).

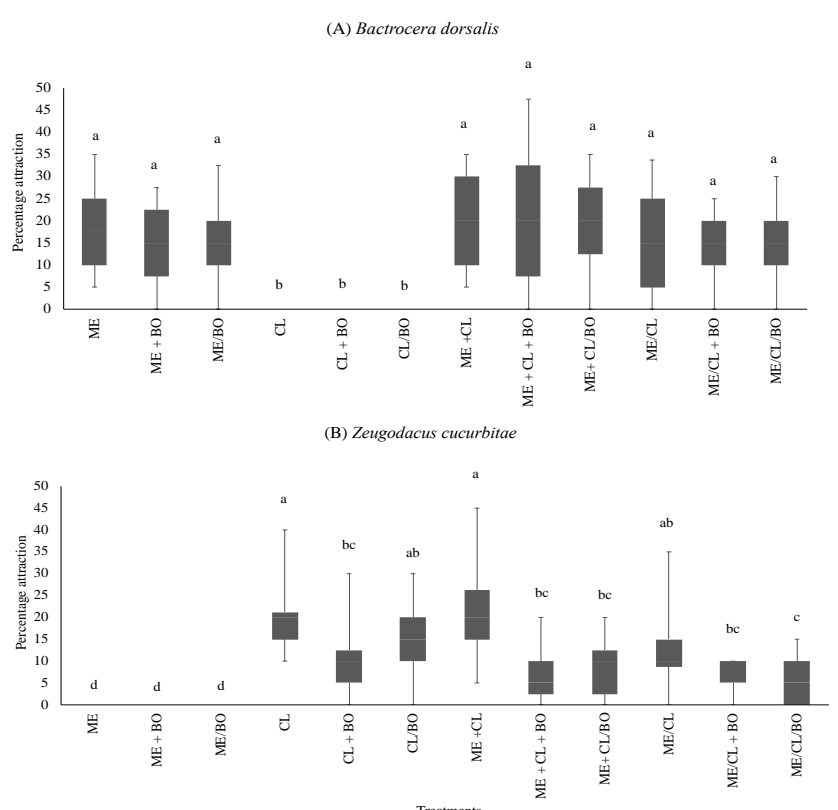

Figure-3. Boxplot analysis of attraction levels of adult Bactrocera dorsalis (A) and Zeugodacus cucurbitae (B) to rubber foam with methyl eugenol (ME) and single cue lure (CL) or mixed lures with and without basil oil (BO) injected at the same $(+\mathrm{BO})$ or a different spot $(/ \mathrm{BO})$.

Table-1. Percentages of attraction of adult male Bactrocera dosalis and Zeugodacus cucurbitae to methyl eugenol (ME) and a single cue lure $(C L)$ or mixed lure $(M E+C L)$ under laboratory conditions.

\begin{tabular}{|c|c|c|c|c|c|c|}
\hline \multirow{2}{*}{ Tested day } & \multirow{2}{*}{$\begin{array}{c}\text { Fruit fly's } \\
\text { Species }\end{array}$} & \multicolumn{5}{|c|}{ Mean percentage of attraction (mean) \pm SE) $)^{\mathbf{1}}$} \\
\cline { 3 - 7 } & B. dorsalis & ME & CL & ME+CL & ME/CL & Control \\
\hline \multirow{2}{*}{0} & Z. cucurbitae & $0.0 \pm 0.0 \mathrm{c}$ & $17.5 \pm 1.4 \mathrm{ab}$ & $27.5 \pm 4.8 \mathrm{a}$ & $13.8 \pm 2.4 \mathrm{~b}$ & $0.0 \pm 0.0 \mathrm{c}$ \\
\cline { 2 - 7 } & B. dorsalis & $30.0 \pm 2.0 \mathrm{a}$ & $0.0 \pm 0.0 \mathrm{~b}$ & $26.3 \pm 2.4 \mathrm{a}$ & $25.0 \pm 3.5 \mathrm{a}$ & $0.0 \pm 0.0 \mathrm{~b}$ \\
\hline \multirow{3}{*}{15} & Z. cucurbitae & $0.0 \pm 0.0 \mathrm{c}$ & $18.8 \pm 2.4 \mathrm{ab}$ & $22.5 \pm 3.2 \mathrm{a}$ & $8.8 \pm 3.1 \mathrm{~b}$ & $0.0 \pm 0.0 \mathrm{c}$ \\
\hline \multirow{2}{*}{30} & B. dorsalis & $15.0 \pm 2.0 \mathrm{a}$ & $0.0 \pm 0.0 \mathrm{~b}$ & $16.3 \pm 2.4 \mathrm{a}$ & $17.5 \pm 1.4 \mathrm{a}$ & $0.0 \pm 0.0 \mathrm{~b}$ \\
\cline { 2 - 7 } & Z. cucurbitae & $0.0 \pm 0.0 \mathrm{~b}$ & $28.8 \pm 4.3 \mathrm{a}$ & $31.3 \pm 4.7 \mathrm{a}$ & $18.8 \pm 6.9 \mathrm{a}$ & $0.0 \pm 0.0 \mathrm{~b}$ \\
\hline \multirow{2}{*}{45} & B. dorsalis & $17.5 \pm 4.3 \mathrm{a}$ & $0.0 \pm 0.0 \mathrm{~b}$ & $18.8 \pm 5.2 \mathrm{a}$ & $8.8 \pm 3.8 \mathrm{a}$ & $0.0 \pm 0.0 \mathrm{~b}$ \\
\cline { 2 - 7 } & Z. cucurbitae & $0.0 \pm 0.0 \mathrm{c}$ & $20.0 \pm 2.0 \mathrm{a}$ & $15.0 \pm 2.0 \mathrm{ab}$ & $11.3 \pm 2.4 \mathrm{~b}$ & $0.0 \pm 0.0 \mathrm{c}$ \\
\hline
\end{tabular}

${ }^{1 /}$ Different letters in the same row indicate statistically significant differences by Tukey' HSD test $(p<0.01)$. 
Table-2. Percentage of attraction of adult male Bactrocera dorsalis and Zeugodacus cucurbitae to rubber foam injected with basil oil (BO) in the same spot with methyl eugenol (ME) and a single cue lure (CL) or mixed lure (ME+CL or ME/CL) under laboratory conditions.

\begin{tabular}{|c|c|c|c|c|c|c|}
\hline \multirow{2}{*}{ Tested day } & \multirow{2}{*}{$\begin{array}{c}\text { Fruit fly's } \\
\text { species }\end{array}$} & \multicolumn{5}{|c|}{ Mean percentage of attraction $($ mean $) \pm \mathrm{SE})^{1 /}$} \\
\hline & & ME+BO & CL+ BO & $\mathrm{ME}+\mathrm{CL}+\mathrm{BO}$ & $\mathrm{ME} / \mathrm{CL}+\mathrm{BO}$ & Control \\
\hline \multirow{2}{*}{0} & B. dorsalis & $5.0 \pm 2.9 \mathrm{a}$ & $0.0 \pm 0.0 \mathrm{a}$ & $1.7 \pm 1.7 \mathrm{a}$ & $3.3 \pm 1.7 \mathrm{a}$ & $0.0 \pm 0.0 \mathrm{a}$ \\
\hline & Z. cucurbitae & $0.0 \pm 0.0 \mathrm{a}$ & $6.7 \pm 1.7 \mathrm{a}$ & $8.3 \pm 4.4 \mathrm{a}$ & $6.7 \pm 3.3 \mathrm{a}$ & $0.0 \pm 0.0 \mathrm{a}$ \\
\hline \multirow{2}{*}{15} & B. dorsalis & $18.3 \pm 7.3 \mathrm{a}$ & $0.0 \pm 0.0 \mathrm{~b}$ & $28.3 \pm 7.3 \mathrm{a}$ & $18.3 \pm 6.0 \mathrm{a}$ & $0.0 \pm 0.0 \mathrm{~b}$ \\
\hline & Z. cucurbitae & $0.0 \pm 0.0 \mathrm{c}$ & $13.3 \pm 1.7 \mathrm{a}$ & $6.7 \pm 1.7 b$ & $8.3 \pm 1.7 \mathrm{ab}$ & $0.0 \pm 0.0 \mathrm{c}$ \\
\hline \multirow{2}{*}{30} & B. dorsalis & $20.0 \pm 7.6 \mathrm{a}$ & $0.0 \pm 0.0 \mathrm{~b}$ & $16.7 \pm 6.0 \mathrm{a}$ & $23.3 \pm 4.4 \mathrm{a}$ & $0.0 \pm 0.0 \mathrm{~b}$ \\
\hline & Z. cucurbitae & $0.0 \pm 0.0 \mathrm{a}$ & $5.0 \pm 5.0 \mathrm{a}$ & $3.3 \pm 1.7 \mathrm{a}$ & $3.3 \pm 1.7 \mathrm{a}$ & $0.0 \pm 0.0 \mathrm{a}$ \\
\hline \multirow{2}{*}{45} & B. dorsalis & $13.3 \pm 1.7 \mathrm{a}$ & $0.0 \pm 0.0 \mathrm{~b}$ & $13.3 \pm 3.3 \mathrm{a}$ & $15.0 \pm 5.0 \mathrm{a}$ & $0.0 \pm 0.0 \mathrm{~b}$ \\
\hline & Z. cucurbitae & $0.0 \pm 0.0 \mathrm{~b}$ & $15.0 \pm 7.6 \mathrm{a}$ & $6.7 \pm 4.4 \mathrm{ab}$ & $6.7 \pm 1.7 \mathrm{ab}$ & $0.0 \pm 0.0 \mathrm{~b}$ \\
\hline
\end{tabular}

${ }^{1 /}$ Different letters in the same row indicate statistically significant differences by Tukey' HSD test $(p<0.01)$.

Table-3. Percentage of attraction of adult male Bactrocera dorsalis and Zeugodacus cucurbitae to rubber foam injected with basil oil (BO) in the different spot with methyl eugenol (ME) and a single cue lure (CL) or mixed lure (ME+CL or ME/CL) under laboratory conditions.

\begin{tabular}{|c|c|c|c|c|c|c|}
\hline \multirow{2}{*}{ Tested day } & \multirow{2}{*}{$\begin{array}{c}\text { Fruit fly's } \\
\text { species }\end{array}$} & \multicolumn{5}{|c|}{ Mean percentage of attraction $($ mean $) \pm \mathrm{SE})^{1 /}$} \\
\hline & & ME/BO & CL/BO & $\mathrm{ME}+\mathrm{CL} / \mathrm{BO}$ & ME/CL/BO & Control \\
\hline \multirow{2}{*}{0} & B. dorsalis & $3.3 \pm 3.3 \mathrm{a}$ & $0.0 \pm 0.0 \mathrm{a}$ & $3.3 \pm 1.7 \mathrm{a}$ & $8.3 \pm 4.4 \mathrm{a}$ & $0.0 \pm 0.0 \mathrm{a}$ \\
\hline & Z. cucurbitae & $0.0 \pm 0.0 \mathrm{~b}$ & $18.3 \pm 1.7 \mathrm{a}$ & $3.3 \pm 3.3 \mathrm{ab}$ & $6.7 \pm 4.4 \mathrm{ab}$ & $0.0 \pm 0.0 \mathrm{~b}$ \\
\hline \multirow{2}{*}{15} & B. dorsalis & $18.3 \pm 10.1 \mathrm{ab}$ & $0.0 \pm 0.0 \mathrm{~b}$ & $23.3 \pm 3.3 \mathrm{a}$ & $13.3 \pm 6.0 \mathrm{ab}$ & $0.0 \pm 0.0 \mathrm{~b}$ \\
\hline & Z. cucurbitae & $0.0 \pm 0.0 \mathrm{~b}$ & $15.0 \pm 2.9 \mathrm{a}$ & $6.7 \pm 4.4 \mathrm{ab}$ & $1.7 \pm 1.7 \mathrm{~b}$ & $0.0 \pm 0.0 \mathrm{~b}$ \\
\hline \multirow{2}{*}{30} & B. dorsalis & $16.7 \pm 1.7 \mathrm{~b}$ & $0.0 \pm 0.0 \mathrm{c}$ & $28.3 \pm 1.7 \mathrm{a}$ & $26.7 \pm 1.7 \mathrm{a}$ & $0.0 \pm 0.0 \mathrm{c}$ \\
\hline & Z. cucurbitae & $0.0 \pm 0.0 \mathrm{a}$ & $6.7 \pm 4.4 \mathrm{a}$ & $6.7 \pm 3.3 \mathrm{a}$ & $5.0 \pm 2.9 \mathrm{a}$ & $0.0 \pm 0.0 \mathrm{a}$ \\
\hline \multirow{2}{*}{45} & B. dorsalis & $15.0 \pm 5.0 \mathrm{a}$ & $0.0 \pm 0.0 \mathrm{~b}$ & $15.0 \pm 2.9 \mathrm{a}$ & $10.0 \pm 2.9 \mathrm{a}$ & $0.0 \pm 0.0 \mathrm{~b}$ \\
\hline & Z. cucurbitae & $0.0 \pm 0.0 \mathrm{~b}$ & $15.0 \pm 2.9 \mathrm{a}$ & $13.3 \pm 3.3 \mathrm{a}$ & $8.3 \pm 1.7 \mathrm{a}$ & $0.0 \pm 0.0 \mathrm{~b}$ \\
\hline
\end{tabular}

${ }^{1 /}$ Different letters in the same row indicate statistically significant differences by Tukey' HSD test $(p<0.01)$.

\section{Efficiency of the mixed lure and basil oil in field condition}

The present study also determined the mean captured fruit flies at fifteen-day intervals for six months. Throughout the trapping period, the ME alone trapped a higher number of $B$. dorsalis than the other treatments, and the trend for trapping was higher during the first three collection dates. The greatest number of $B$. dorsalis captured were in the second collection date $(22.5 \pm 5.0$ FTD $)$ with a highly significant variation $\left(\mathrm{F}_{6,21}=74.124 ; p<0.001\right)$, followed by the ME/BO trap (14.4 \pm 1.4 FTD) (Figure 4A). Upon the fourth collection date, the number of files obtained in each treatment was not significantly different. A fluctuating trend of trapping was observed between the location where $\mathrm{ME}$ alone was always higher, followed by either $\mathrm{ME} / \mathrm{BO}$ or $\mathrm{ME}+\mathrm{CL} / \mathrm{BO}$ with significant variations (Figure 4A).

Conversely, the trapping trend for Z. cucurbitae was higher during the first three consecutive collection dates with highly significant variations $\left(\mathrm{F}_{6,21}=4.149\right.$; $p<0.001)$. The ME+CL trap caught the greatest number of $Z$. cucurbitae followed by CL (Figure 4B). At the third collection date $(0.8 \pm 0.3$ FTD $)$ the highest number of flies was found in the $\mathrm{ME}+\mathrm{CL}$ trap. Afterwards, fluctuations in the trend of trapping by CL and $\mathrm{ME}+\mathrm{CL}$ were observed. The combination of $\mathrm{CL}$ and $\mathrm{ME}+\mathrm{CL}$ with basil oil showed a reduction in the number of trapped flies in comparison to $\mathrm{CL}$ and $\mathrm{ME}+\mathrm{CL}$ without basil oil, which were just slightly higher than that of the control (Figure 4B).

Boxplot analyses of trapped flies from the $2^{\text {nd }}$ week to the $24^{\text {th }}$ week (six months) were performed. For $B$. dorsalis, $\mathrm{ME}$ alone and $\mathrm{ME} / \mathrm{BO}$ were not significantly different, ranging from 3.8-5.5 FTD; whereas CL alone and $\mathrm{CL} / \mathrm{BO}$ did not attract $B$. dorsalis (Figure 5A). For Z. cucurbitae, CL alone and ME+CL showed the same number of trapped flies with $0.3 \pm 0.03$ and $0.3 \pm 0.06 \mathrm{FTD}$, respectfully. CL alone and the $\mathrm{ME}+\mathrm{CL}$ combination with $\mathrm{BO}$ presented a decrease in 
the number of trapped flies with $0.1 \pm 0.02$ and $0.2 \pm$ 0.05 FTD, respectfully (Figure 5B). Z. cucurbitae were not detected in $\mathrm{ME}, \mathrm{ME} / \mathrm{BO}$, or the control trap. In our field study, we used single lures (ME and CL) and mixed (ME+CL), single lures mixed with basil oil $(\mathrm{ME} / \mathrm{BO}, \mathrm{CL} / \mathrm{BO})$, and mixed lures $(\mathrm{ME}+\mathrm{CL} / \mathrm{BO})$. We assumed that because basil oil contains ME, the basil oil inclusions would increase the effectiveness of the traps; however, our results found that the most efficient trap was the single ME trap, which was similarly matched by the ME+CL traps. The greatest mean number of flies was achieved with the ME traps, followed by the $\mathrm{ME}+\mathrm{CL}$ and $\mathrm{ME} / \mathrm{BO}$ traps. It could be that the ME in basil oil somehow reduced the attraction of ME.

Interestingly, $\mathrm{ME}+\mathrm{CL}$ outperformed the $\mathrm{ME} / \mathrm{BO}$ traps, as the mix of the two lures proved more attractive to $\mathrm{CL}$ responsive species than those responsive to ME. In the case of $B$. dorsalis, the ME trap was the most attractive. For $Z$. cucurbitae, the $\mathrm{ME}+\mathrm{CL}$ trap was equally effective as the CL trap, whereas the CL and ME+CL traps with basil oil were less effective than the traps without basil oil. These results suggest that basil oil may inhibit CL activity.

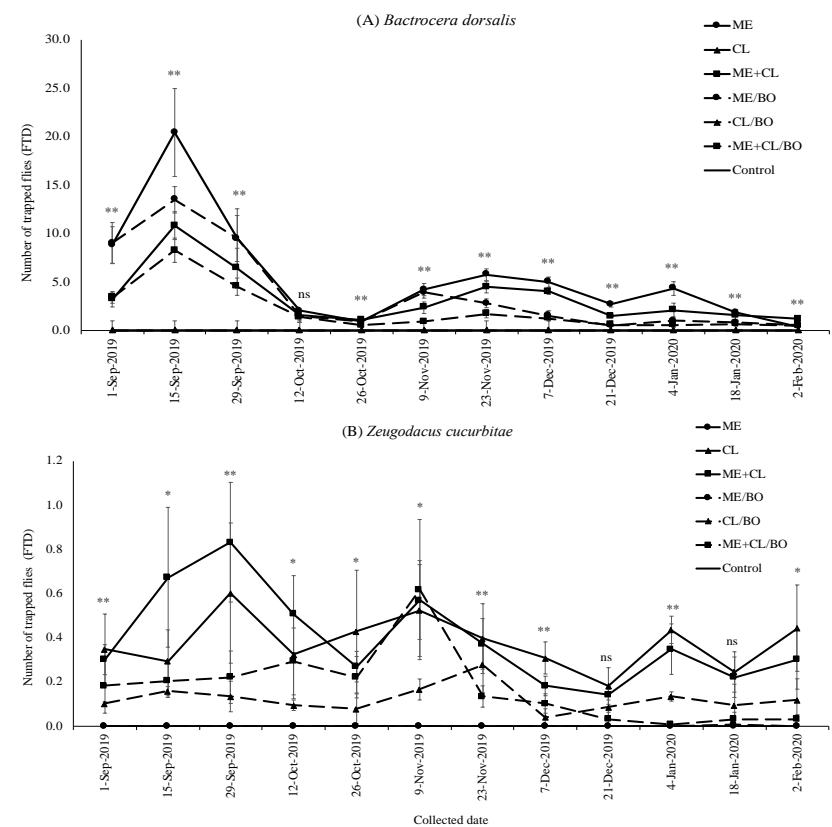

Figure-4. Mean number of trapped flies (FTD) of Bactrocera dorsalis (A) and Zeugodacus cucurbitae (B) collected every two weeks from the field up to the $24^{\text {th }}$ week ( 6 months).
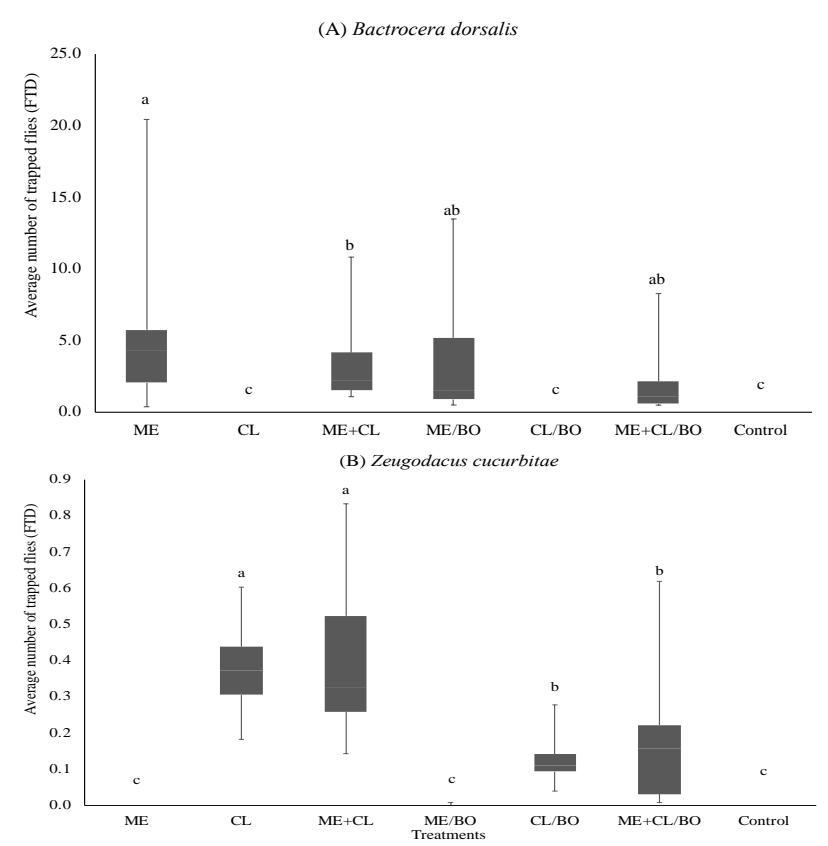

Figure-5. Boxplot analysis of the mean number of trapped flies (FTD) of Bactrocera dorsalis (A) and Zeugodacus cucurbitae (B) within each lure treatment under field conditions.

\section{Discussion}

The insecticidal activity of the essential oils basil, clove, citronella, and eucalyptus were injected in the para-rubber foam against $B$. dorsalis and $Z$. cucurbitae and tested, thereby increasing the number of possible essential oils to combat tephritid fruit flies. The present study evaluated the toxicity of these essential oils, and determined that eucalyptus oil was the most toxic, causing $100 \%$ mortality within 12 hours (Day 0 ); followed by clove, basil, and citronella oil for both $B$. dorsalis and Z. cucurbitae. This general finding is supported by other studies with similar results. Eucalyptus oil proved the most poisonous against the peach fruit fly $B$. zonata (Sanders) pupa $\left(\mathrm{LC}_{50}\right.$ value $38.88 \mathrm{ml} / \mathrm{L}$ ), followed by basil and other essential oils (Ali, 2018). Hidayat (2014) demonstrated the strong fumigant effects on the first and second instar larvae of the Queensland fruit fly, B. tryoni (Froggatt). However, our experiments utilized varied exposure times to the EOs within the rubber foam receptacles and proved no longer useful for both types of flies after extended weathering. The major constituents of eucalyptus oil are monoterpene and sesquiterpene hydrocarbons (Russo et al., 2015). As we have 
observed, eucalyptus oil was found to be more active in a raw form immediately after exposure.

Basil oil proved the most effective against $B$. dorsalis, more so than $Z$. cucurbitae, after weathering for 30 days. Similar results were found with basil (Ocimum basilicum L.) oil, which has three major active structures (trans-anethole, estragole, and linalool) against $B$. dorsalis and $Z$. cucurbitae. The toxic action of basil oil in $C$. capitata occurred significantly faster than in $Z$. cucurbitae, yet moderately faster in $B$. dorsalis (Chang et al., 2009). Among Tephritid fruit flies, the Mediterranean fruit fly is the most investigated species involving interactions with essential oils. When tea tree (Melaleuca alternifolia, Myrtaceae) oil was applied in contact and fumigation assay to $C$. capitata and its parasitoid Psyttalia concolor, the highly toxic results produced a lower $\mathrm{LC}_{50}$ value towards $C$. capitata over its parasitoid $P$. concolor [contact: $0.117 \mu \mathrm{l} \mathrm{oil} / \mathrm{cm}^{2}$ vs $0.470 \mu \mathrm{l}$ $\mathrm{oil} / \mathrm{cm}^{2}$; fumigation: $2.239 \mu \mathrm{loil} / \mathrm{L}$ air vs $9.348 \mu \mathrm{loil} / \mathrm{L}$ air] (Benelli et al., 2013). In their earlier study, essential oils from fresh rosemary, lavender, chan, poiteau, and arborvitae leaves were imposed upon $C$. capitata and lavender oil proved to be the most toxic at 24 hours $\left(\mathrm{LC}_{50}\right.$ of $9.09 \mu \mathrm{g} / \mathrm{L}$ ) (Benelli et al., 2012). Kostyukovsky et al. (2002) determined that some plant essential oils act very quickly and effectively on various insect pests by affecting the neuromodulator octopaminergic. This receptor is absent in mammals, making such actions less hazardous to other non-target organisms and the environment.

Essential clove oil was also found to have a toxic effect on fruit flies of both species. For B. dorsalis, death rates were $60.0 \%$ and $87.5 \%$ after 72 hours on Day 0 and Day 15, respectively. For Z. cucurbitae, decreased mortality rates of 36.7 to $40.0 \%$ were observed after 72 hours on Days 0 and 15. Further studies in EO toxicity were conducted against Mexican fruit flies Anastrepha ludens (Loew). Essential oils from Eugenia caryophyllus, Ocimum basilicum, and Thymus vulgaris were used to determine the ingestion toxicity against adult $A$. ludens; in which $E$. caryophyllus proved the most toxic with $\mathrm{LC}_{50} 3,529$ ppm, followed by $T$. vulgaris $(5,347 \mathrm{ppm})$ and $O$. basilicum (8,050 ppm) (Buentello-Wong et al., 2016). Notably, these EOs were also toxic to $B$. dorsalis and $Z$. cucurbitae under laboratory conditions.

We incorporated basil oil (BO) within the male lures, $\mathrm{ME}$, and CL; at the same and different positions in the rubber foam, and observed their levels of attraction. However, satisfactory results were found only in the single lures. The addition of BO with ME did not increase the effectiveness of ME alone in attracting $B$. dorsalis; and, with CL, a reduction in the attraction of Z. cucurbitae was observed. Chang et al. (2009) determined that a mixture of $\mathrm{ME}$ and $\mathrm{BO}$ did not affect the toxicity of BO to B. dorsalis. They surmised the ME may play a physiological role in toxicity reduction. Excessive concentrations of CL (50 and 90 $\%$ ) in combination with $\mathrm{BO}$ decreased the mortality or knockdown in $Z$. cucurbitae males and females. Additionally, a mixture of linalool with CL showed decreased potency to the species as concentrations of CL increased.

Dharmadasa et al. (2015) used two morphotypes of Ocimum tenuiflorum (L.) to attract B. dorsalis and compared the results with those of ME. Their results focused on the mean number of flies attracted in the first few weeks (53-111 flies), but did not involve mixing plant essential oils with lures. In our experiments with $B$. dorsalis, single $\mathrm{ME}$ and $\mathrm{ME}$ mixed with $\mathrm{BO}$ showed no effect on fly attraction. For Z. cucurbitae, the CL and CL mixed with $\mathrm{BO}$ showed a relative decrease in attraction. A mix of $\mathrm{CL}$ and $\mathrm{BO}$ would, therefore, have an antagonistic effect.

Chang et al. (2013) developed a single package of $\mathrm{ME}+\mathrm{BO}$ with a modified area wide pest management (AWPM) standard trap for B. dorsalis. They observed that it took two hours for flies entering the traps to die. Their results also indicated that the $\mathrm{ME}+\mathrm{BO}$ formulation was effective for 90 days and beyond. They observed differences in the two studies due to the amounts of BO within each dispenser. They found that mortality increased with added amounts in the sponge dispensers (size $7.5 \mathrm{~cm} \times 5 \mathrm{~cm}$ ) that contained 5,000 $\mu \mathrm{l}$ of ME:BO $(1,000 \mu \mathrm{l}: 4,000 \mu \mathrm{l})$. In contrast, the rubber foam (size $5 \mathrm{~cm} \times 2 \mathrm{~cm}$ ) utilized in the study herein contained $400 \mu \mathrm{l}$ of ME:BO $(100 \mu \mathrm{l}$ : $300 \mu \mathrm{l})$. They further observed that only the ME trap caught a greater number of $B$. dorsalis than the $\mathrm{ME}+\mathrm{BO}$ trap and the control.

\section{Conclusion}

Among the four plant essential oils tested, BO proved the most toxic within the first 15-30 days. The single $\mathrm{ME}$ and mixture of ME with $\mathrm{BO}$ were similar in the attraction of $B$. dorsalis, but for $Z$. cucurbitae the mixture of $\mathrm{CL}$ with $\mathrm{BO}$ decreased attraction. In the field tests, the single ME performed similarly to the combination of $\mathrm{ME}$ and $\mathrm{BO}$ in trapping $B$. dorsalis, whereas the combination of CL with $\mathrm{BO}$ presented an 
antagonistic effect in trapping Z. cucurbitae. We may, therefore, conclude that a trap with $\mathrm{ME}$ and $\mathrm{BO}$ may be an effective alternative for the $B$. dorsalis management in organic farming systems, further leading to a reduction of chemical insecticides in the environment.

\section{Acknowledgment}

We would like to thank Thailand's Education Hub for the Southern Region of ASEAN Countries, (Contract No. TEH-AC 020/2018), the Center of Excellence in Agricultural and Natural Resources Biotechnology: Phase 2, and the Agricultural Innovation and Management Division, Faculty of Natural Resources, Prince of Songkla University for their financial support and the use of their facilities. We also wish to acknowledge the copy-editing services of RDO/PSU and the helpful guidance of Dr. Seppo Karrila.

Disclaimer: None.

Conflict of Interest: None.

Source of Funding: This research was supported by the government budget of Prince of Songkla University, grant number NAT590861S.

\section{References}

Ali MA, 2018. Toxicity of certain plant oils on pupil stage of the peach fruit fly, B. zonata (Sunders) (Tephritidae: Diptera) Adv. Plants. Agric. Res. 8: 372-374.

Arancibia M, Rabossi A, Bochicchio PA, Moreno S, López Caballero ME, Gómez Guillén M DC and Montero P, 2013. Biodegradable films containing clove or citronella essential oils against the Mediterranean fruit fly Ceratitiscapitata (Diptera: Tephritidae). J. Agric. Food. Tech. 3: 1-7.

Aketarawong N, Guglielmino CR, Karam N, Falchetto M, Manni M, Scolari F and Malacrida AR, 2014. The oriental fruit fly Bactrocera dorsalis ss in East Asia: disentangling the different forces promoting the invasion and shaping the genetic make-up of populations. Genetica 142: 201-213.

Benelli G, Flamini G, Canale A, Cioni PL and Conti B, 2012. Toxicity of some essential oil formulations against the Mediterranean fruit fly Ceratitiscapitata

(Wiedemann)(Diptera:Tephritidae). Crop Protection 42:223-229.
Benelli G, Flamini G, Fiore G, Cioni PL and Conti B, 2013. Larvicidal and repellent activity of the essential oil of Coriandrum sativum L. (Apiaceae) fruits against the filariasis vector Aedes albopictus Skuse (Diptera: Culicidae). Parasitol. Res. 112: 1155-1161.

Buentello-Wong S, Galán-Wong L, Arévalo-Niño K, Almaguer-Cantú V and Rojas-Verde G, 2016. Toxicity of some essential oil formulations against the Mexican fruit fly Anastrepha ludens (Loew) (Diptera: Tephritidae). Ind. Crops. Prod. 85: 5862.

Calvert KO, 1982. Polymer latices and their applications. London: Applied Science.

Canale A, Benelli G, Conti B, Lenzi G, Flamini G, Francini A and Cioni PL, 2013. Ingestion toxicity of three Lamiaceae essential oils incorporated in protein baits against the olive fruit fly, Bactrocera oleae (Rossi) (DipteraTephritidae). Nat. Prod. Res. 27: 2091-2099.

Chang CL, Cho IK, Li QX, Manoukis NC and Vargas RI, 2013. A potential field suppression system for Bactrocera dorsalis Hendel. J. Asia-Pac. Entomol. 16:513-519.

Chang CL, Kyu Cho I and Li QX, 2009. Insecticidal activity of basil oil, trans-anethole, estragole, and linalool to adult fruit flies of Ceratitis capitata, Bactrocera dorsalis, and Bactrocera cucurbitae. J. Econ. Entomol. 102:203-209.

Desneux N, Decourtye A and Delpuech JM, 2007. The sublethal effects of pesticides on beneficial arthropods. Annu. Rev. Entomol. 52: 81-106.

Dharmadasa RM, Siriwardhane DAS, Samarasinghe K, Rangana SHCS, Nugaliyadda L, Gunawardane I and Aththanayake AML, 2015. Screening of two Ocimum tenuiflorum L. (Lamiaceae) morphotypes for their morphological characters, essential oil composition and fruit fly attractant ability. World J. Agric. Res. 3: 1-4.

Drew RA and Romig MC, 2013. Tropical fruit flies (Tephritidae:Dacinae) of South-East Asia: Indomalaya to North-West Australasia. CABI.

Hidayat Y, 2014 Biological activities of essential and vegetable oils against Queensland fruit fly Bactrocera tryoni (Froggatt) (Diptera: Tephritidae). PhD Thesis, School of Agriculture and Food Sciences, The University of Queensland, Australia.

Ilyas A, Khan HAA, and Qadir A, 2017. Effect of Essential Oils of some Indigenous Plants on Settling and Oviposition Responses of Peach Fruit 
Fly, Bactrocera zonata (Diptera: Tephritidae). Pakistan J. Zool. 49: 1547-1553.

Isman MB and Machial CM, 2006. Pesticides based on plant essential oils: from traditional practice to commercialization. pp. 29-44. In M. Rai and M. Carpinella (eds.), Naturally occurring bioactive compounds. Advances in phytomedicine, vol. 3. Elsevier Science, Amsterdam, Switzerland.

Kéita SM, Vincent C, Schmit JP, Ramaswamy S and Bélanger A, 2000. Effect of various essential oils on Callosobruchus maculatus (F.) (Coleoptera: Bruchidae). J. Stored Prod. Res. 36: 355-364.

Kostyukovsky M, Rafaeli A, Gileadi C, Demchenko N and Shaaya E, 2002. Activation of octopaminergic receptors by essential oil constituents isolated from aromatic plants: possible mode of action against insect pests. Pest Manag. Sci. 58: 11011106.

López SB, López ML, Aragón LM, Tereschuk ML, Slanis AC, Feresin GE, Zygadlo JA and Tapia AA, 2011. Composition and anti-insect activity of essential oils from Tagetes L. species (Asteraceae, Helenieae) on Ceratitis capitata Wiedemann and Triatoma infestans Klug. J. Agr. Food Chem. 59: 5286-5292.

Maggi F and Benelli G, 2018. Essential oils from aromatic and medicinal plants as effective weapons against mosquito vectors of public health importance, pp. 69-129. In G. Benelli and H. Mehlhorn (eds.), Mosquito-borne diseases: implications for public health. Springer International Publishing, New York, United States.

Malee R, 2019. Product development of natural rubber foam mixed with pheromone, methyl eugenol, for attractant Oriental fruit fly, Bactrocera dorsalis (Hendel) (Diptera: Tephritidae). Dissertation, Prince of Songkla University, Songkhla, Thailand.

Metcalf RL and Metcalf ER, 1992. Fruit flies of the family Tephritidae, pp. 109-152. In R.L. Metcalf and E.R. Metcalf (eds.), Plant kairomones in insect ecology and control. Springer, New York, United States.

Prabhakar CS, Mehta PK, Sood P, Singh SK, Sharma $\mathrm{P}$ and Sharma PN, 2012a. Population genetic structure of the melon fly, Bactrocera cucurbitae (Coquillett) (Diptera: Tephritidae) based on mitochondrial cytochrome oxidase (COI) gene sequences. Genetica. 140: 83-91.
Prabhakar CS, Sood P and Mehta K, 2012b. Pictorial keys for predominant Bactrocera and Dacus fruit flies (Diptera: Tephritidae) of north western Himalaya. Arthropods. 1(3): 101-111.

Qin Y, Krosch MN, Schutze MK, Zhang Y, Wang X, Prabhakar CS, Susanto A, Hee AKW, Ekise S, Badji K, Khan M, Wu J, Wang Q, Zhu L, Zhao Z, Liu L, Clarke AR and Li Z, 2018. Population structure of a global agriculture invasive pest, Bactrocera dorsalis (Diptera: Tephritidae). Evol. Appl. 11: 1990-2003.

Rossi YE and Palacios SM, 2015. Insecticidal toxicity of Eucalyptus cinerea essential oil and 1, 8cineole against Musca domestica and possible uses according to the metabolic response of flies. Ind. Crop. Prod. 63: 133-137.

Royer JE and Mayer DG, 2017. Combining cue-lure and methyl eugenol in traps significantly decreases catches of most Bactrocera, Zeugodacus and Dacus species (Diptera: Tephritidae: Dacinae) in Australia and Papua New Guinea. J. Econ. Entomol. 111(1): 298-303.

Russo Cabrera N, Chludil H, Yeber-Grass $M$ and Leicach S, 2015. Insecticidal activity of young and mature leaves essential oil from Eucalyptus globulus Labill. Against Tribolium confusum Jacquelin du Val (Coleoptera: Tenebrionidae). Chil. J. Agric. Res. 75: 375-379.

SPSS, 2001. SPSS for Windows 11. SPSS Inc., Chicago (IL) URL http://www. spss.com.

Vargas RI, Stark JD, Kido MH, Ketter HM and Whitehand LC, 2000. Methyl eugenol and cuelure traps for suppression of male oriental fruit flies and melon flies (Diptera: Tephritidae) in Hawaii: effects of lure mixtures and weathering. J. Econ. Entomol. 93(1): 81-87.

\section{Contribution of Authors}

Akter MM: Conceived idea, designed research methodology, data collection, data analysis and manuscript writing.

Theary K: Data collection and analysis.

Kalkornsurapranee E: Manuscript final reading and approval.

Prabhakar CS: Designed research methodology, manuscript final reading and approval. Thaochan N: Conceived and designed the research, manuscript editing, statistical analysis. 